\title{
Comparaison des conduites discursives de mères et d'enseignants dans la co-construction de récits avec des enfants
}

\author{
Vinel Elise \\ Université Sorbonne Nouvelle (Sorbonne Paris Cité)- CLESTHIA \\ Elise.vinel@gmail.com
}

\section{Introduction}

Cette communication s'intéresse aux conduites discursives produites par des mères et des enseignants au cours de deux lectures conjointes d'album avec des enfants. Elle présente une description et une analyse de la manière dont les adultes racontent des histoires aux enfants et de l'expérience spécifique des récits qu'ils leur offrent.

Avant l'âge de 6 ans, les enfants ne sont pas en mesure de raconter seuls une histoire bien construite. Raconter nécessite de se représenter mentalement les objets, les personnages, les événements décrits dans le texte, mais aussi de construire une chaîne causale qui relie les différentes actions du ou des personnages aux buts poursuivis, même si d'autres aspects doivent être pris en compte. Or, ces compétences se construisent non seulement au contact des récits oraux ou écrits, des livres rencontrés mais aussi autour de leurs interactions avec des adultes plus experts. Selon cette perspective interactionniste, initiée par Vygotski (1997) puis Bruner (1983), l'expérience discursive des enfants joue un rôle essentiel dans l'acquisition de toute compétence langagière et donc en particulier des compétences narratives. Ainsi, les conduites de récit que les adultes offrent aux enfants sont un élément majeur dans la construction de leurs conduites de récit futures. Mais cette expérience des récits n'est pas homogène chez tous les enfants : certains sont confrontés à tous types d'histoires (avec texte ou sans texte), alors que d'autres ne sont confrontés qu'à des récits oraux, des livres documentaires ou à des scripts, ou encore à des histoires vues à la télévision (Sénéchal, Lefèvre \& Daley, 1998 ; Sulzby \& Teale, 1991; Mason \& Allen, 1986). Cette expérience débute très tôt dans l'environnement quotidien des enfants, bien avant d'apprendre à lire et à écrire (Bruner, 1996). Les enfants qui entrent à l'école arrivent donc avec des expériences diverses de l'écrit qui peuvent s'avérer être bien différentes de celles proposées à l'école. Il nous paraît alors intéressant de comparer et de saisir les différentes expériences de récit offertes aux enfants.

De nombreuses études ont été menées sur les effets des lectures conjointes. Ces lectures peuvent permettre d'attirer l'attention des enfants sur les caractéristiques particulières du livre (Ninio \& Bruner, 1978) mais aussi les amener à saisir des aspects fondamentaux de la lecture (Snow et Ninio, 1986). Pour ces dernières auteures, à travers les lectures conjointes, les parents aident leurs enfants à comprendre que les livres servent à lire et non à être simplement manipulés, que les images des livres correspondent à des représentations de choses et d'événements, qu'elles peuvent être nommées, que les événements décrits sont fictionnels et se produisent donc en dehors du temps réel.

Mais une grande majorité des travaux sur les lectures conjointes se sont axés principalement sur la littératie émergente. Sulzby (1985), en particulier, souligne l'importance de l'expérience de la lecture dans le développement de la capacité à raconter puis à lire des histoires mais aussi l'importance des relectures d'une même histoire dans la construction des récits. Son étude compare les tentatives émergentes de lecture d'enfants au début et à la fin de l'école maternelle aussi bien que celles d'enfants âgés de 2, 3 et 4 ans. Elle montre ainsi que les enfants les plus jeunes et les moins expérimentés passent d'un discours peu structuré proche du langage parlé à un discours comportant un registre et une intonation appropriée dans la lecture d'histoires. Les enfants les plus âgés et les plus expérimentés utilisent au fur et à mesure un discours proche de celui de l'auteur. L'auteure en conclut alors que les enfants internalisent les connaissances sur le registre de la langue bien avant qu'ils ne deviennent des lecteurs classiques. Plus 
spécifiquement, ces lectures de livres permettent aux enfants d'être confrontés aux formes grammaticales de la langue écrite et du discours littéraire (Bus, van Ijzendoorn \& Pellegrini, 1995 ; Grossmann, 1999).

D'autres travaux se sont plus particulièrement intéressés aux interactions adultes-enfants. Bus, van Ijzendoorn \& Pellegrini (1995) considèrent notamment que même s'il est nécessaire qu'il y ait des livres dans l'environnement des enfants, que les enfants voient leurs parents lire, qu'ils se rendent régulièrement à la bibliothèque, qu'ils lisent de temps à autre des histoires avec leurs parents, cela ne s'avère pas suffisant. Il est primordial que les adultes interagissent autour de ces histoires avec les enfants. Certaines recherches ont cherché en particulier à comprendre les processus d'interaction mis en place au cours de la lecture et les stratégies utilisés par les adultes (Pellegrini, Brody, \& Sigel, 1985; Sulzby \& Teale, 1991; Senechal, Lefèvre \& Daley, 1998). Certaines d'entre elles (Low \& Durkin, 2001 ; Reese \& Fivush, 1993) montrent que les mères qui utilisent un langage riche et élaboré lors de lectures conjointes d'histoires augmentent la capacité de leurs enfants à raconter des histoires et leur permettent d'employer par ailleurs un langage riche et élaboré. De plus, Reese \& Fivush (1993) soulignent que les mères qui évoquent régulièrement des expériences vécues passées avec leurs enfants permettent non seulement à ces derniers d'utiliser plus facilement le passé dans leurs discours mais aussi améliorent les compétences narratives de leurs enfants.

Snow (1983) puis Thomas (1985) mettent également en avant le rôle prépondérant des processus d'interaction dans l'apprentissage du langage des enfants. Elles considèrent que lorsque les adultes prennent en compte les assertions des enfants, les poursuivent, ajoutent de nouvelles informations, répondent à leurs questions, leur en posent, mais aussi structurent le dialogue qu'ils engagent avec eux, évitent de les mettre en échec, les responsabilisent quant à la difficulté de la tâche, l'apprentissage, entre autres, de la compréhension des récits en est facilité. Il est par conséquent primordial que soient instaurées, selon ces auteures, une qualité et une quantité importante d'interactions autour de lectures conjointes.

Toutes ces études soulignent donc l'importance de l'acculturation précoce à l'écrit et des médiations des adultes notamment à travers la lecture conjointe de livres pour un meilleur apprentissage de la lecture. Cependant, comme le constate à juste titre Grossmann (2001), écouter une histoire écrite nécessite une capacité d'attention particulière, mais aussi une capacité à intégrer les informations, ou à relier les segments textuels entre eux. Ces différentes compétences ne viennent pas naturellement aux enfants, il est nécessaire que les adultes les aident à les construire. Grâce à leur expérience des livres et de la langue écrite les adultes vont permettre aux enfants d'accéder à une meilleure compréhension de la langue écrite. Or, cet apprentissage ne se fait pas par simple contact ou imprégnation, il n'est possible que si l'enfant participe activement aux échanges. L'adulte ne construit donc pas seul le récit au cours de la lecture des albums, il le co-construit avec les enfants. Chaque participant occupe alors au cours de cette lecture dialoguée une place particulière qui peut varier en fonction de l'intérêt de chacun pour la tâche ou des difficultés rencontrées.

Comme le soulignent Rondal (1983) mais aussi Danis, Bernard et Leproux (2000), l'adulte s'adapte à son interlocuteur. Ainsi, de manière générale, selon Rondal, lorsque l'adulte s'adresse à un enfant, il peut modifier certains aspects phonologiques, sémantiques, syntaxiques ou encore fonctionnels de son langage. Il réduit, en particulier, sa diversité lexicale, utilise plus de mots à référence concrète, ajuste la longueur moyenne de ses énoncés à un niveau légèrement supérieur à celle de l'enfant. Il a également tendance à produire des énoncés grammaticalement bien formés, sans grande complexité syntaxique, utilise un nombre important d'interrogatives, suivies des impératives et des déclaratives. Il produit ainsi de nombreuses requêtes d'information, requêtes d'action et descriptions et réalise un nombre important d'autorépétitions, de feedback (approbations, répétitions avec adjonctions, corrections explicites).

Lors des lectures conjointes, Ninio and Bruner (1978) constatent que les mères proposent à l'enfant un modèle, un rituel de comportements. Elles posent des questions, fournissent des renseignements, produisent des feedback en répétant ou en élargissant les remarques des enfants et mettent ainsi en place un discours étayant. Elles permettent donc à l'enfant à travers ces «formats» (Bruner, 1983) de construire un récit sans lesquels il n'y serait pas parvenu. 
Parmi les conduites des adultes, de nombreuses études anglo-saxones (McNamee, 1987 ; Pratt, Kerig, Cowan \& Cowan, 1988 ; Nelson, 1991 ; Peterson \& McCabe, 1994) mais aussi françaises (Florin, 1991 ; Lafontaine, 1984 ; Rondal, 1978) soulignent l'importance des questions dans le discours des adultes. Ces questions permettent non seulement d'établir et de maintenir la communication verbale entre l'adulte et l'enfant (Snow, 1978) mais aussi de développer les capacités langagières de l'enfant en lui donnant l'occasion de parler et de s'affirmer comme partenaire dans le dialogue (Lafontaine, 1984 ; Danis, Bernard, \& Leproux, 2000) ou encore de favoriser chez lui un langage plus sophistiqué (Pelligrini et al., 1985; Sénéchal et al., 1995). Les études françaises de Lafontaine (1984) et Rondal (1978) montrent notamment que $36 \%$ à $49 \%$ des énoncés des adultes correspondent à des questions et la première, en particulier, que les adultes posent des questions courtes aux enfants, quel que soit leur âge, variées dans leurs formes et leurs fonctions. De plus, certaines structures et mots interrogatifs apparaissent plus fréquemment que d'autres dans le discours des adultes : les questions en « oui/non » sont ainsi légèrement plus importantes que les questions commençant par les interrogatifs du type « où », «quoi », « comment », «que ». Dans ces deux études les questions en « oui/non » représentent environ $20 \%$ des questions alors que les autres types de questions entre de 10 à $25 \%$.

De plus, Danis et al. (2000) mais aussi McGinty, Justice, Zucker, Gosse \& Skibbe (2012) remarquent qu'il existe non seulement une influence de l'input parental sur les capacités cognitives des enfants mais aussi une influence des réactions des enfants dans les productions des adultes. McGinty et al. montrent en particulier en quoi les questions des mères peuvent influencer le niveau de participation verbale des enfants mais aussi, inversement, en quoi le niveau de participation verbale des enfants influence les questions posées par les mères lors de lectures conjointes.

Pour vérifier si les compétences narratives des enfants émergent d'interactions sociales avec les adultes McNamee (1987) a demandé à des enfants âgés de 5 à 6 ans de rappeler une même histoire à leur enseignant au cours de trois sessions différentes. Lors du premier rappel, les enfants n'ont donné seuls que quelques éléments de récit. Leurs enseignants les ont beaucoup guidés et ont rappelé une grande partie de l'histoire. Ensuite, lors du second rappel, les enseignants ont aidé les enfants à raconter l'histoire en leur posant des questions en «wh- » permettant ainsi aux enfants de trouver les dernières informations dont ils avaient besoin pour raconter l'histoire (questions du type : «qu'est-ce que le roi fait quand la jeune fille dit non ?») ou en leur fournissant des informations sous la forme de tag-questions (tagquestions du type : "Il partit voir le père de la jeune fille, n'est-ce pas ? »). Lors du troisième rappel, les enfants ont été capables, seuls, de mieux raconter l'histoire. McNamee en conclut que les enfants, à travers le guidage des enseignants, ont appris et intériorisé un certain nombre de compétences nécessaires au rappel d'histoire.

Pratt et al. (1988) ont observé, quant à eux, comment des parents d'enfants de 3 ans se sont comportés dans une tâche de rappel de récits d'histoires. Ils ont mis en évidence que les enfants répondaient davantage aux questions fournissant déjà une information (questions du type : "Le vélo est-il cassé ? ») qu'aux questions trop générales (questions du type : «Peux-tu me raconter ton histoire? »).

Ces deux dernières études montrent ainsi que les feedback des adultes sous forme de questions en vue d'obtenir des informations sur des événements spécifiques de l'histoire permettent d'augmenter la quantité, la qualité et la richesse des récits d'histoire des enfants. D'autres études, par ailleurs, constatent que lorsque des parents ou d'autres adultes posent des questions spécifiques de type " qui », " quand », « pourquoi », « où » et « comment» au cours de l'histoire racontée et qu'ils développent les réponses des enfants, ces derniers établissent des connexions entre différents éléments de l'histoire et améliorent ainsi la construction et le rappel des histoires (Nelson, 1991; Peterson \& McCabe, 1994).

Toutes ces études montrent ainsi l'importance des lectures conjointes et des interactions entre adultes et enfants, à la maison ou à l'école, et plus particulièrement le rôle primordial du questionnement dans le développement des compétences narratives des enfants. Cependant, la plupart des études menées se focalisent sur un type de récit, le livre avec texte, et ne comparent pas les différentes expériences discursives proposées par les mères et les enseignants au cours de ces interactions. 
C'est en partant de ce cadre que nous nous interrogeons sur les différentes conduites discursives produites par les adultes, mères et enseignants, avec des enfants, à partir de lectures dialoguées d'albums sans texte puis avec texte, en milieu scolaire et familial. Nous cherchons ainsi, d'une part, à comparer ces différentes conduites discursives offertes aux enfants, à travers l'étude de leurs interventions verbales et plus particulièrement de leur questionnement, et d'autre part, à analyser comment les adultes étayent le discours des enfants à travers leurs questions. Ceci nous amène alors à chercher à comprendre et à établir s'il existe une influence du milieu (scolaire vs familial), des participants au dialogue (enfants cibles, pairs, parents, enseignants), de l'âge des enfants ou encore du type de support (albums avec texte vs albums sans texte) dans l'utilisation des conduites discursives des adultes et plus particulièrement des questions mais aussi si peuvent intervenir des profils types de conduites des adultes comme l'ont notamment suggéré Salazar Orvig et de Weck (à paraître).

\section{Méthodologie}

Nous commencerons par présenter le corpus et les livres illustrés proposés aux différents participants, puis nous détaillerons les types d'interventions verbales produites par les adultes, plus particulièrement les questions qu'ils adressent aux enfants et pour finir, parmi ces dernières celles qui jouent un rôle étayant.

\subsection{Corpus et livres illustrés}

Notre corpus ${ }^{1}$, composé des enregistrements de 10 enfants ( 5 filles et 5 garçons) âgés de 3 à 5 ans ( 3 enfants en Petite Section, 4 enfants en Moyenne Section et 3 enfants en Grande Section), a été recueilli dans une école maternelle parisienne ainsi qu'au domicile des enfants. Les mères observées exercent toutes une activité professionnelle ( 5 employées et 5 cadres), le père observé est cadre. Deux des enseignants avaient six ans d'ancienneté, le troisième en avait 30 au moment de l'enregistrement. Chaque enfant a été filmé à quatre reprises : lors de deux séances de lecture dialoguée d'un album avec un enseignant et des pairs, puis lors de deux autres séances de lecture, seul avec sa mère. Un enfant a été ajouté aux neuf autres de Moyenne Section car un de ces enfants parlait très peu en classe, cependant il a été filmé pour la lecture sans texte avec sa mère et la lecture avec texte avec son père. A l'école comme à la maison, la première séance de lecture correspondait à un album sans texte, puis la seconde à un album avec texte. Les groupes étaient composés de 11 enfants en Petite Section, de 10 en Moyenne Section et de 12 en Grande Section. La durée des interactions était libre et se prolongeait le temps de l'échange. Les séances à l'école pour l'album sans texte ont duré chacune en moyenne $27 \mathrm{mn}$ et pour l'album avec texte environ $20 \mathrm{mn}$. A la maison, en revanche, pour l'album sans texte, la durée a pu varier de $5 \mathrm{mn} 15 \mathrm{sec}$, pour la séance la plus courte, à $26 \mathrm{mn}$ pour la plus longue avec une durée moyenne de $15 \mathrm{mn} 15 \mathrm{sec}$. Pour l'album avec texte, les séances ont été globalement homogènes, et ont donc duré en moyenne $10 \mathrm{mn}$, sauf une de $20 \mathrm{mn}$. La consigne, dans toutes ces situations, était de raconter l'histoire ensemble comme ils avaient l'habitude de faire. Les deux livres illustrés sans texte retenus pour cette étude ont été : "Ah les belles vacances des petits cochons" de Goodall, pour les mères et "Le voleur de poule" de Rodriguez, pour les enseignants. Deux livres illustrés avec texte ont également été lus : "La grotte de Petit Ours" de Waddell pour l'ensemble des mères, "Le bateau de Petit Ours" de Bunting pour les enseignants de Petite Section et Grande Section. Un troisième livre a dû être également proposé. L'enseignant de Moyenne Section a fait la lecture de "Toi et moi, Petit Ours" de Waddell car un des enfants avait déjà lu celui proposé aux Petite Section et Grande Section.

\subsection{Transcriptions et analyses}

Les enregistrements ont été transcrits sous CLAN ${ }^{2}$ puis codés par des chercheurs et des codeurs entraînés selon des critères élaborés par le groupe de recherche du projet DIAREF. Chaque tour de parole a été décomposé en énoncés caractérisés selon leur valeur illocutoire. Nous parlerons ainsi d'interventions. 
Afin de répondre aux questions que nous nous sommes posées nous allons, dans un premier temps, nous intéresser aux interventions verbales produites par les adultes dans les différents contextes en se focalisant plus spécifiquement sur les différents types de questions posées, puis nous analyserons parmi ces questions celles qui sont étayantes.

\subsubsection{Types d'interventions verbales des adultes}

Afin de situer la place qu'occupent les questions dans le dialogue, nous allons commencer par nous intéresser à l'ensemble des interventions verbales produites par les adultes. Pour caractériser ces interventions nous nous basons sur les catégories élaborées dans le cadre du projet FNRS suisse «Interactions mère-enfant en situation logopédique $»^{3}$ dirigé par de Weck. Elles sont au nombre de sept :

- Les assertions (ASS) : ce sont toutes les assertions sauf les réponses aux questions

- Les assertions sollicitantes (ASO) : elles se situent entre les assertions et les questions et ont la particularité de contenir un élément sollicitant du type «tu sais», «tu vois», « hein», « regarde »; elles peuvent ou non engendrer une réaction de la part de l'enfant

- Les réponses (REP) : ce sont des réactions sous forme d'assertions à la suite de questions ou d'assertions sollicitantes (les auto-réponses aux questions auto-adressées sont considérées comme des assertions)

- Les questions (QUE) : elles sollicitent une conduite discursive telle que les demandes d'information, de clarification, de confirmation, d'explication, de narration, les propositions, les ébauches, les relances

- Les ordres (ORD) : ils correspondent aux demandes ou requêtes d'action ou injonctions de conduite verbale

- Les autres (AUT) : ils équivalent aux acquiescements, refus, phatiques ou exclamations

- Les indécidables (INDC) : pour les cas de verbal incompréhensible, d'énoncés inachevés ou pour tout autre intervention que le contexte ne permet pas de catégoriser

Dans notre corpus, pour la lecture sans texte, à l'école, les interventions verbales sont au nombre de 3555 avec une variation allant de 1040 à 1266 et à la maison au nombre de 4922 avec une variation allant de 340 à 1689. Pour la lecture avec texte, nous avons exclu des interventions verbales les interventions correspondant à de la lecture. Ainsi, le nombre d'interventions verbales, à l'école, est de 1813 avec une variation allant de 479 à 672 , et à la maison de 2371 avec une variation allant de 55 et 603 .

\subsubsection{Types de questions adressées aux enfants}

Les questions occupent une place importante dans les types d'interventions verbales et s'avèrent primordiales pour la construction du récit, nous avons donc cherché à déterminer les caractéristiques des questions effectuées par les différents adultes. Pour cela, nous nous appuyons sur les travaux de François et al. (1984), de Weck (1998) et Rosat (1998), et nous en dégageons trois grandes catégories :

- $\quad$ Les questions totales (QT), parmi lesquelles nous trouvons :

○ Les questions fermées (QF) : auxquelles la réponse se fait par « oui » ou par «non »

- Les questions alternatives (QA) : qui offrent une alternative pour la réponse (« tu crois que c'est son papa ou sa maman ?») 
- Les questions partielles $(\mathrm{QP})$, parmi lesquelles nous trouvons :

- Les ébauches (EBAU) : lorsque la formulation de l'énoncé préfigure la catégorie de la réponse («pour avancer c'est une..+ ? »)

- Les questions catégorielles $(\mathrm{QC})$ : qui induisent une catégorie linguistique (questions commençant par « qui », « quoi », « où », « quand »)

- Les questions ouvertes (QO), parmi lesquelles nous trouvons :

- Les questions en «pourquoi/comment» (QPC) : qui n'induisent pas nécessairement la forme de la réponse

- Les questions portant sur les événements (QE) du type « que se passe-t-il ? », « qu'estce qu'ils vont faire? »

○ Les relances (REL) du type « hein? », « et puis? », « alors? », « oui Paul ? » qui sont isolées. Quand ces particules suivent immédiatement une question elles sont codées comme cette dernière.

Nous avons fait le choix de placer les questions en «pourquoi/comment» dans la catégorie des questions ouvertes car elles ne préfigurent pas la forme de la réponse.

\subsubsection{Rôle des questions étayantes adressées aux enfants}

Pour que l'enfant puisse parvenir à construire seul des récits il va s'appuyer sur l'expérience que les adultes en ont et plus particulièrement sur les conduites discursives que ces derniers mettent en place pour produire le récit. Parmi ces conduites discursives le questionnement joue un rôle essentiel. Nous nous intéressons alors plus particulièrement aux questions étayantes que posent les adultes.

Nous nous appuyons sur les travaux de Bruner (1983), d'Hudelot \& Vasseur (1997), de Weck (1998) mais aussi plus particulièrement sur ceux d'Ingold et al. (2008) puis Bignasca \& Rezzonico (2010) pour catégoriser les différents types d'étayage. Ces derniers auteurs distinguent notamment deux types d'étayage: un étayage verbal linguistique, qui soutient la production de l'enfant au niveau de l'élaboration linguistique et un étayage verbal spécifique de la tâche qui permet à l'enfant de participer activement à l'accomplissement de la tâche en cours.

Les questions portant sur l'étayage linguistique de la tâche sont de différents types :

- des demandes de dénomination :

Exemple 1

MER JUL $58 a$

et c'est quoi ça et ça?

Exemple 2

ENS MS 214a on appelle comment ce chemin ça?

\section{- des demandes de clarification :}

Exemple 3

MER LIL 24a il quoi? 
Exemple 4
ENS MS $158 a$
comment ça pourquoi les poules elles vont venir?

- des demandes de définition :

Exemple 5

MER ELI $13 a \quad$ c'est quoi une petite caverne?

Exemple 6

ENS GS $119 b$

un autre mot pour dire courir après?

- des ébauches qui peuvent porter sur des éléments narratifs ou des dénominations :

Exemple 7

MER JAM $18 b$

un plu(meau $)+$. ?

Exemple 8

ENS PS $130 a$

parce qu'il y a la lune et les $+\ldots$ ?

L'étayage de la tâche se décline en catégories plus spécifiques qui se traduisent également par différents types de questions (Bignasca \& Rezzonico, 2010), auxquels nous avons ajouté un dernier type, les demandes d'implication dans la tâche. Ainsi, nous avons :

- Le guidage : l'adulte amène l'enfant à participer à la narration en lui adressant des questions factuelles (sur les éléments de la page qui font l'objet de l'attention conjointe de la dyade), des questions anticipatrices (portant sur la suite de l'histoire et les pages du livre qui vont suivre), des demandes d'explication (à caractère non descriptif et de type " pourquoi " ou " comment "), ainsi que des relances (incitations non spécifiques permettant l'avancement de l'activité).

Exemple 9

MER ANA $74 c$

où est ce qu'il va avec ses beaux habits?

Exemple 10

ENS PS $227 a \quad$ pourquoi il l'a donné à Petit Ours?

- Les conduites réflexives : l'adulte propose à l'enfant, à travers ses questions, un modèle qui rend explicite son raisonnement en relation avec l'activité en cours. Cela peut se traduire par des demandes d'approbation par rapport à ses interprétations et à ses dires, par des questions fermées anticipant sur la suite ou revenant sur des événements antérieurs de l'histoire ou qui appartiennent à la connaissance partagée de la dyade. 
Exemple 11

Exemple 12

ENS MS 111a est-ce que vous croyez que Grand Ours va le trouver?

- Les réactions de l'adulte aux conduites de l'enfant : elles ont pour but d'évaluer, d'accepter ou de refuser les propositions de ce dernier, en lui fournissant un feed-back. Parmi ces dernières, nous n'avons retenu que les questions qui soutiennent l'enfant dans l'élaboration de la narration (ex : réactions sous forme d'évaluation positive, de confrontation ou d'interventions de synthèse des contributions de l'enfant ou de la dyade).

Exemple 13
MER JEA $80 a$
il se débat tu crois?

Exemple 14

ENS PS $52 a \quad$ tu crois qu'il peut se noyer quand il est dans la barque?

- Les demandes d'implication dans la tâche : elles sollicitent l'attention de l'enfant, veillent à son maintien, l'enrôlent dans la tâche et lui permettent de s'y impliquer (ex : « tu vois ?», «tu reconnais », « tu comprends », etc.)

Exemple 15

MER OSC $48 \mathrm{c} \quad$ tu t(e) souviens d(e) ça?

Exemple 16

ENS GS $128 b \quad$ vous le savez? \%act: TEA montre de nouveau l'image aux enfants

Partant de là, nous allons observer les conduites des adultes, mères et enseignants, lors de la construction des divers récits avec les enfants.

\section{Résultats}

Nous nous attacherons dans un premier temps à analyser les interventions verbales des adultes au cours du dialogue, puis nous chercherons à caractériser les questions qu'ils posent puis plus particulièrement celles qui étayent l'activité et permettent ainsi une meilleure participation de l'enfant. 


\subsection{Types d'interventions verbales des adultes}

\subsubsection{Types d'interventions verbales présents dans la lecture sans texte}

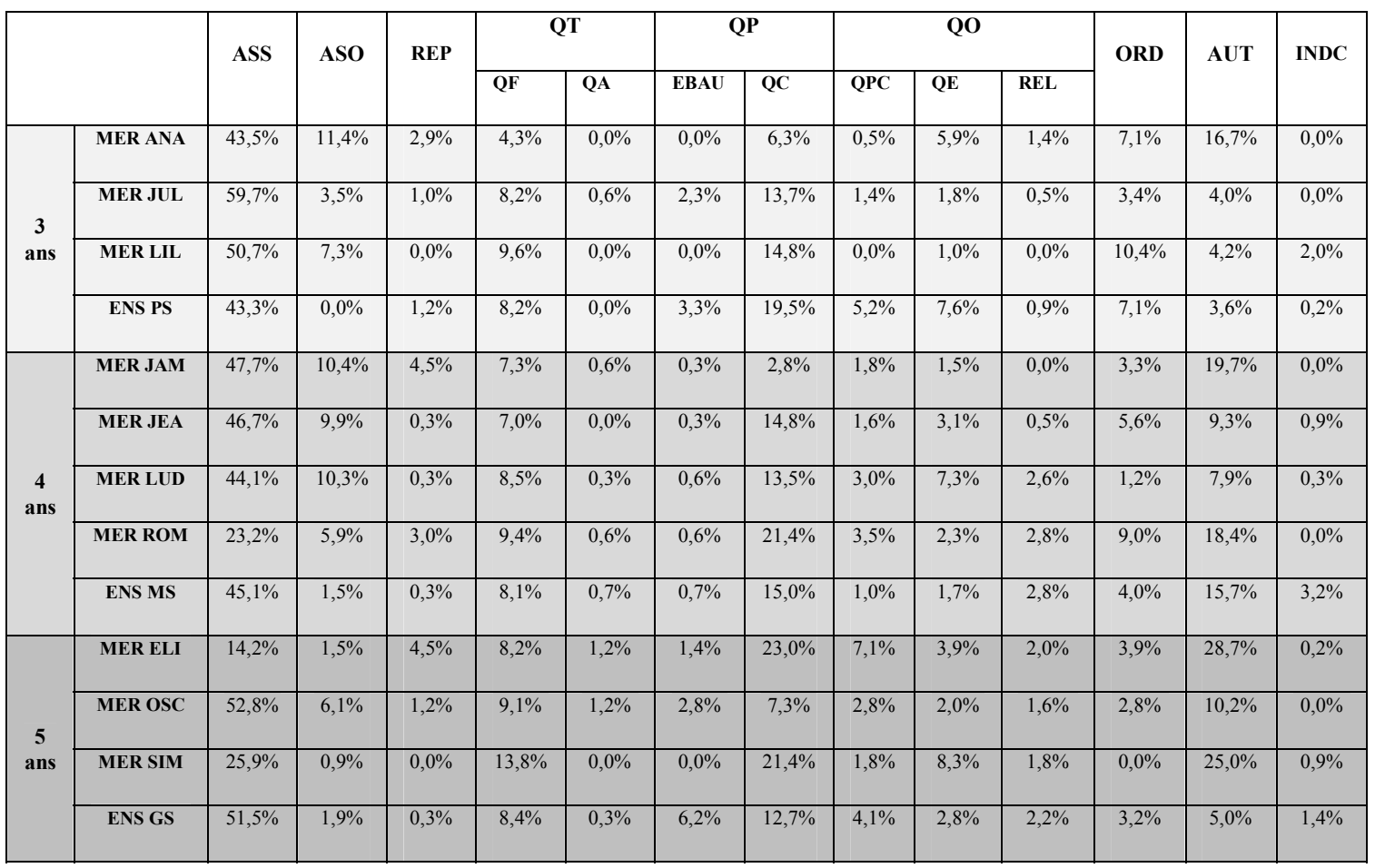

Tableau 1 : Types d'interventions verbales des adultes, par classe d'âge, dans la lecture sans texte

Pour la lecture sans texte, les types d'interventions verbales qui prédominent chez les adultes sont les assertions et les questions.

A l'école, les assertions sont plus présentes que les questions dans le discours des enseignants de Moyenne Section et de Grande Section alors que leur nombre est quasiment identique dans celui de l'enseignant de Petite Section. En outre, ce dernier recourt plus aux questions que les deux autres alors que celui de Grande Section produit beaucoup plus d'assertions que les enseignants de Petite et Moyenne Section.

Observons ce qui se passe pour l'enseignant de Petite Section qui se montre plus questionnant que les deux autres. Sont présentés en particulier les types de question et les types d'étayage de l'adulte auxquels nous ferons par la suite référence.

Exemple 17

ENS PS118: $\quad b$ mais que se passe-t-il pour les personnages qui sont là l'ours le lapin et le coq? \%act: ENS pointe du doigt les personnages sur l'image

ENF JUL41:

ENF ROM36:

ENS PS119:

$<i(l)$ s s'arrêtent $>[>]$.

ENS PS119:

$<i(l)$ s sont fatigués $>[<]$.

ils ont l'air < fatigués $>[>]$. 


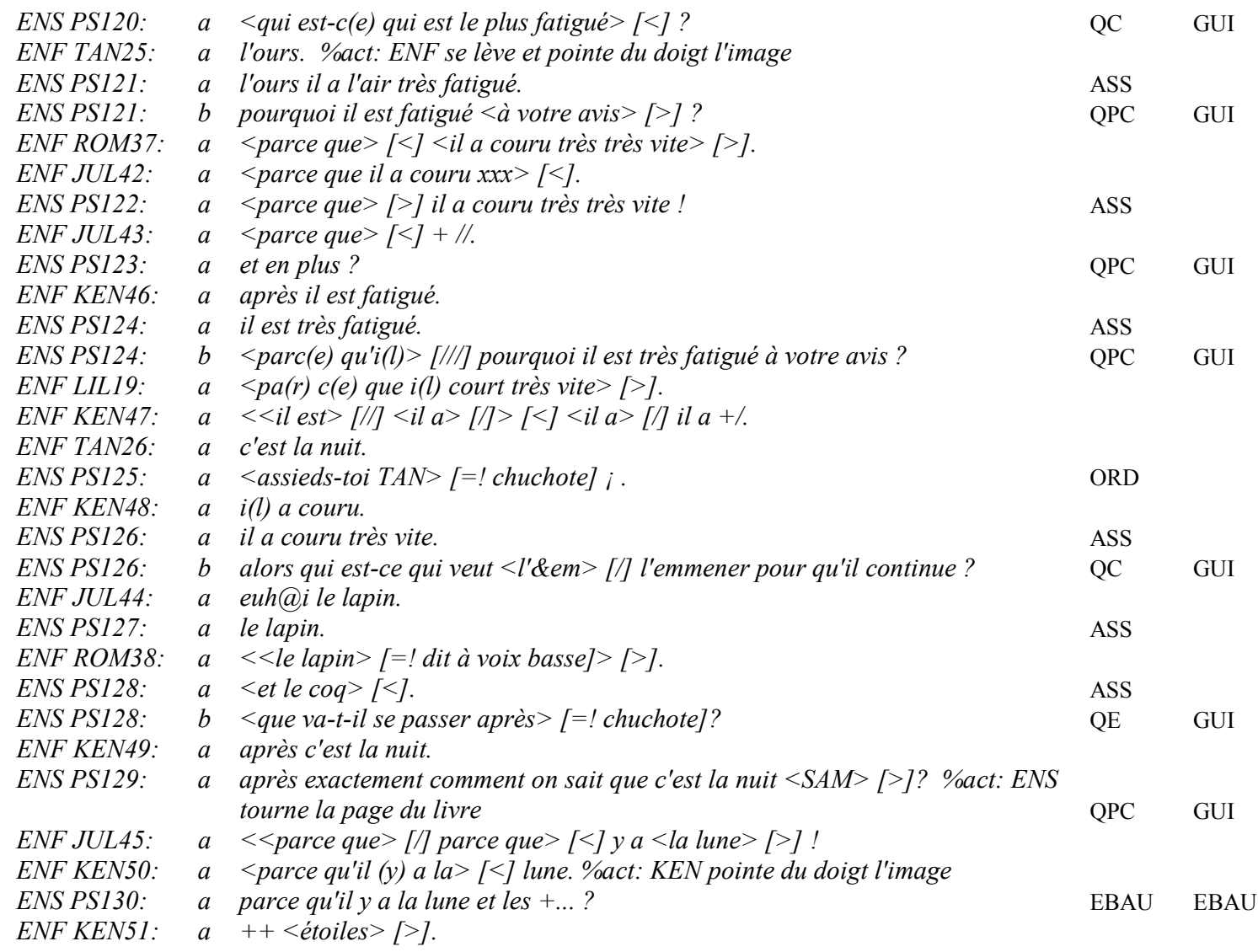

Dans cet exemple, nous voyons bien que l'enseignant mène le dialogue à la fois à partir de questions et d'assertions. Les questions semblent cependant davantage faire progresser la construction du récit que les assertions qui paraissent plus dédiées à reprendre et reformuler les énoncés des enfants.

A la maison, les conduites des mères diffèrent quelque peu. Les mères des enfants de 3 ans (ANA, JUL et LIL) comme trois des mères des enfants de 4 ans (JAM, JEA et LUD) produisent nettement plus d'assertions que de questions. Une des mères des enfants de 4 ans (ROM) et deux des mères des enfants de 5 ans (ELI et SIM) interviennent, à l'inverse, davantage à partir de questions que d'assertions. Les interventions verbales d'une mère d'un enfant de 5 ans (OSC) semblent atypiques par rapport à celles deux autres mères de cette tranche d'âge puisqu'elle produit plus d'assertions cependant, ces interventions s'approchent de celles de l'enseignant de Grande Section.

Observons plus précisément ce qui se passe chez une mère d'un enfant de 4 ans qui se présente comme moins questionnante que les autres.

\section{Exemple 18}

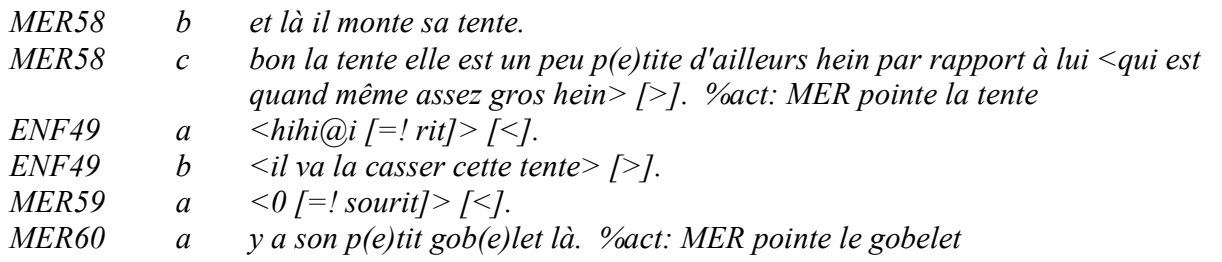




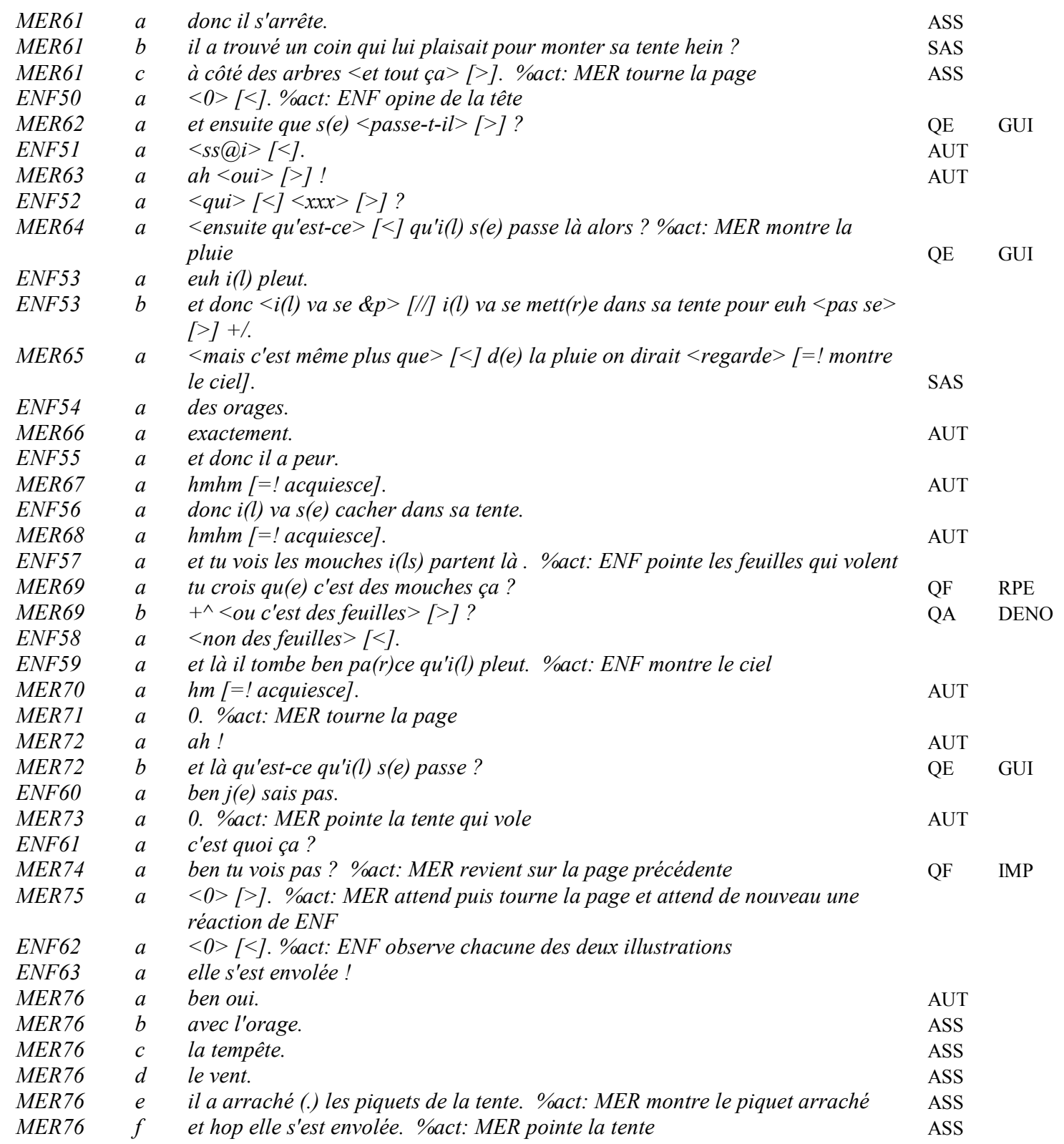

Dans cet exemple, nous voyons bien l'emprise des assertions sur les questions. La mère de JAM utilise fortement les assertions pour décrire ce qui se passe ou ce qui est présent sur les images et dans une nettement moindre mesure les questions.

Par ailleurs, pour l'ensemble des adultes, la présence plus ou moins importante des autres types d'interventions verbales (les réponses, les ordres, les assertions sollicitantes et les « autres ») semblent dépendre essentiellement de variables individuelles. Les assertions sollicitantes s'avèrent, en revanche, davantage produites par les mères que par les enseignants, quel que soit l'âge des enfants. 


\subsubsection{Types d'interventions verbales présents dans la lecture avec texte}

\begin{tabular}{|c|c|c|c|c|c|c|c|c|c|c|c|c|c|c|}
\hline & \multirow{2}{*}{ ASS } & \multirow{2}{*}{ ASO } & \multirow{2}{*}{ REP } & \multicolumn{2}{|c|}{ QT } & \multicolumn{2}{|c|}{$\overline{Q P}$} & \multicolumn{3}{|c|}{$\overline{\text { QO }}$} & \multirow{2}{*}{ ORD } & \multirow{2}{*}{ AUT } & \multirow{2}{*}{ INDC } \\
\hline & & & & & $\overline{Q F}$ & $\overline{Q A}$ & EBAU & $\overline{\mathrm{QC}}$ & $\overline{\text { QPC }}$ & $\overline{Q E}$ & REL & & & \\
\hline \multirow{4}{*}{$\begin{array}{c}3 \\
\text { ans }\end{array}$} & MER ANA & $27,3 \%$ & $7,8 \%$ & $4,5 \%$ & $15,4 \%$ & $0,0 \%$ & $0,0 \%$ & $11,1 \%$ & $1,4 \%$ & $5,2 \%$ & $1,2 \%$ & $8,5 \%$ & $17,6 \%$ & $0,0 \%$ \\
\hline & MER JUL & $59,7 \%$ & $4,5 \%$ & $3,7 \%$ & $8,3 \%$ & $0,8 \%$ & $0,4 \%$ & $7,6 \%$ & $1,2 \%$ & $0,7 \%$ & $0,5 \%$ & $2,8 \%$ & $9,9 \%$ & $0,0 \%$ \\
\hline & MER LIL & $32,9 \%$ & $9,5 \%$ & $0,0 \%$ & $35,1 \%$ & $0,0 \%$ & $0,0 \%$ & $9,1 \%$ & $3,4 \%$ & $1,9 \%$ & $0,0 \%$ & $0,0 \%$ & $5,6 \%$ & $2,5 \%$ \\
\hline & ENS PS & $45,5 \%$ & $1,7 \%$ & $1,8 \%$ & $4,4 \%$ & $0,4 \%$ & $3,9 \%$ & $21,5 \%$ & $6,2 \%$ & $6,2 \%$ & $0,5 \%$ & $4,6 \%$ & $3,4 \%$ & $0,0 \%$ \\
\hline \multirow{5}{*}{$\begin{array}{c}4 \\
\text { ans }\end{array}$} & MER JAM & $56,2 \%$ & $13,9 \%$ & $3,9 \%$ & $5,4 \%$ & $0,0 \%$ & $1,4 \%$ & $4,5 \%$ & $1,0 \%$ & $1,5 \%$ & $0,0 \%$ & $1,6 \%$ & $10,5 \%$ & $0,0 \%$ \\
\hline & MER JEA & $34,0 \%$ & $14,3 \%$ & $1,4 \%$ & $15,2 \%$ & $1,1 \%$ & $0,0 \%$ & $6,1 \%$ & $0,0 \%$ & $2,7 \%$ & $2,8 \%$ & $10,6 \%$ & $11,9 \%$ & $0,0 \%$ \\
\hline & MER LUD & $38,7 \%$ & $23,3 \%$ & $0,0 \%$ & $8,0 \%$ & $0,1 \%$ & $0,0 \%$ & $6,6 \%$ & $1,3 \%$ & $2,8 \%$ & $0,9 \%$ & $3,6 \%$ & $14,8 \%$ & $0,0 \%$ \\
\hline & PER ROM & $32,2 \%$ & $2,9 \%$ & $2,1 \%$ & $12,2 \%$ & $1,8 \%$ & $4,9 \%$ & $16,3 \%$ & $6,1 \%$ & $2,8 \%$ & $0,3 \%$ & $2,5 \%$ & $15,0 \%$ & $1,0 \%$ \\
\hline & ENS MS & $45,7 \%$ & $1,1 \%$ & $1,7 \%$ & $8,2 \%$ & $0,1 \%$ & $1,1 \%$ & $11,9 \%$ & $4,3 \%$ & $2,5 \%$ & $1,2 \%$ & $1,9 \%$ & $19,2 \%$ & $1,2 \%$ \\
\hline \multirow{4}{*}{$\begin{array}{c}5 \\
\text { ans }\end{array}$} & MER ELI & $22,8 \%$ & $11,1 \%$ & $21,7 \%$ & $12,0 \%$ & $0,0 \%$ & $0,0 \%$ & $11,8 \%$ & $1,5 \%$ & $0,0 \%$ & $0,0 \%$ & $1,4 \%$ & $17,7 \%$ & $0,0 \%$ \\
\hline & MER OSC & $22,8 \%$ & $0,0 \%$ & $3,9 \%$ & $13,3 \%$ & $2,3 \%$ & $0,0 \%$ & $2,5 \%$ & $8,0 \%$ & $0,0 \%$ & $2,2 \%$ & $8,3 \%$ & $36,7 \%$ & $0,0 \%$ \\
\hline & MER SIM & $20,5 \%$ & $4,1 \%$ & $0,0 \%$ & $20,7 \%$ & $6,4 \%$ & $0,0 \%$ & $27,2 \%$ & $0,0 \%$ & $0,9 \%$ & $0,0 \%$ & $0,0 \%$ & $20,2 \%$ & $0,0 \%$ \\
\hline & ENS GS & $59,8 \%$ & $4,9 \%$ & $0,1 \%$ & $3,7 \%$ & $0,0 \%$ & $4,7 \%$ & $10,6 \%$ & $1,0 \%$ & $0,1 \%$ & $3,6 \%$ & $0,7 \%$ & $10,2 \%$ & $0,7 \%$ \\
\hline
\end{tabular}

Tableau 2 : Types d'interventions verbales des adultes, par classe d'âge, dans la lecture avec texte

Pour la lecture avec texte, les types d'interventions verbales qui prédominent sont, comme pour la lecture sans texte, les assertions et les questions.

A l'école, les assertions se rencontrent plus dans les discours de tous les enseignants que les questions. Par ailleurs, plus les enfants sont jeunes plus les enseignants produisent des questions et plus les enfants sont âgés plus les enseignants émettent des assertions. Les conduites discursives des enseignants de Petite Section et de Moyenne Section ne varient guère entre les deux types de support, seules celles de l'enseignant de Grande Section changent. En effet, il produit un nombre plus important d'assertions et diminue nettement son nombre de questions.

A la maison, les conduites discursives des mères se montrent beaucoup plus contrastées. Une mère d'un enfant de 3 ans (JUL) et trois mères d'enfants de 4 ans (JAM, JEA et LUD) produisent plus d'assertions que de questions. A l'inverse, deux mères d'enfants de 3 ans (ANA et LIL), le père de l'enfant de 4 ans (ROM) et les trois mères des enfants de 5 ans (ELI, OSC et SIM) privilégient les questions aux assertions. Ainsi, trois mères (ANA, LIL et OSC) inversent leurs conduites discursives d'un support à l'autre alors que les autres les maintiennent mais de manière plus ou moins variée en augmentant ou diminuant leur nombre de questions ou d'assertions. Seule une mère d'un enfant de 5 ans (SIM) ne change guère de conduites.

Par ailleurs, d'un support à l'autre, le nombre d'assertions a légèrement augmenté chez les enseignants et le nombre de questions diminué alors qu'inversement, deux tiers des mères ont diminué leur nombre d'assertions et un tiers a augmenté son nombre de questions. De plus, le nombre d'assertions sollicitantes a augmenté chez deux tiers des adultes. Elles s'avèrent cependant, pour les deux types de support, beaucoup plus importantes chez les mères que chez les enseignants. 
Ainsi, ces constats permettent de mettre en évidence différents points.

Premièrement, pour la lecture sans texte, il semblerait que l'âge des enfants ait une incidence sur le type d'interventions verbales privilégié par les adultes. En effet, les questions sont plus présentes chez l'enseignant de Petite Section et les mères d'enfants plus âgés et les assertions chez les enseignants de Moyenne et Grande Section et les mères d'enfants plus jeunes. Les conduites des mères des enfants de 4 ans se trouvant dans un de ces groupes. Pour la lecture avec texte, ce phénomène se retrouve dans les conduites des enseignants et des mères d'enfants de 5 ans. Les autres mères penchant plus ou moins vers un type d'interventions verbales sans dépendance réelle avec l'âge des enfants.

Deuxièmement, le type de support peut avoir une influence sur le type de conduite des adultes. En effet, même si seulement un quart des adultes (deux enseignants et une mère) ne change pas de conduites discursives (production du nombre d'assertions ou de questions quasi équivalente entre les deux types de support), un quart des adultes (trois mères) adopte des conduites inverses à celles de la lecture sans texte et la moitié des adultes (un enseignant et cinq mères) garde le même type de conduites, mais en amplifiant la dominance des questions ou des assertions.

Troisièmement, il semblerait, plus particulièrement chez les enseignants, que l'album sans texte demande une conduite plus guidée de la narration alors que l'album avec texte engendrerait davantage une coconstruction du récit.

Voyons si ces hypothèses d'influence de l'âge et du type de support se confirment dans le cas des questions.

\subsection{Types de questions adressées aux enfants}

Les types de questions, rappelons-le, sont de trois ordres : les questions totales, les questions partielles et les questions ouvertes. Les premières sont composées de questions fermées et alternatives ; les secondes d'ébauches et de questions catégorielles et les dernières de questions en «pourquoi/comment», de relances et de questions portant sur les événements.

Pour donner un ordre de grandeur du nombre de questions posées au cours du dialogue, nous avons calculé le taux de questions posées par chaque locuteur adulte par minute. Il en ressort que pour la lecture sans texte les enseignants comme les mères posent en moyenne 7 questions par minute, avec une variation chez les mères allant de 4 à 10 questions par minute. Pour la lecture avec texte nous n'avons comptabilisé que les questions posées par l'adulte lui-même et non les questions présentes dans le texte du livre à lire. Il en ressort que les enseignants comme les parents posent en moyenne 4 questions par minute, avec une variation chez les enseignants allant de 3 à 7 questions par minute et chez les parents une variation allant de 2 à 7 questions par minute. Les adultes de notre recherche posent alors moins de questions que ceux faisant partie de la recherche de Florin (1991) qui postule que les enseignants produisent en moyenne 6 questions par minute.

Ces résultats corroborent ceux que nous avons avancés précédemment à savoir que la lecture sans texte demande une conduite plus guidée, plus soutenue par des questions que la lecture avec texte.

\subsubsection{Types de questions posées dans la lecture sans texte}

Par ailleurs, pour la lecture sans texte, parmi les trois grands types de questions établis (questions totales, partielles et ouvertes), les questions partielles prédominent chez plus des trois quart des adultes, tous les enseignants, trois mères d'enfants âgés de trois (ANA, JUL et LIL), trois mères d'enfants de 4 ans (JEA, LUD et ROM) et deux mères d'enfants de 5 ans (ELI et SIM). Deux autres, une d'un enfant de 4 ans (JAM) et une d'un enfant de 5 ans (OSC) émettent plus de questions totales. La première, comme nous avons pu le voir dans l'exemple 18, fournit un grand nombre de questions totales fermées ou alternatives et dans une moindre mesure des questions partielles et ouvertes. 
Par ailleurs, les enseignants de Moyenne Section et Grande Section produisent un plus grand nombre de questions totales par rapport à l'enseignant de Petite Section qui privilégie les questions ouvertes, comme nous l'avons vu dans l'exemple 17 seule une question totale est émise par cet enseignant. Les questions totales de ces enseignants représentent environ $8 \%$ des interventions verbales. Cette répartition s'avère moindre que celle évoquée dans les études de Rondal (1978), Lafontaine (1984) ou encore Florin (1991).

Chez les mères, la répartition des types de questions dépend plus de variables individuelles.

En observant plus attentivement chaque catégorie de questions, il semblerait, en outre, que les questions en "pourquoi/comment» soient en grande majorité plus présentes dans les discours des mères des enfants plus âgés que des plus jeunes. Les questions alternatives et les ébauches s'avèrent globalement minoritaires chez l'ensemble des adultes. Ces dernières sont cependant en grande majorité émises par les enseignants et plus particulièrement par celui de Grande Section. Les relances dépendent de variables individuelles.

\subsubsection{Types de questions posées dans la lecture avec texte}

Pour la lecture avec texte, les enseignants privilégient, de la même manière que pour la lecture sans texte, les questions partielles aux autres types de questions. Les enseignants de Moyenne et Grande Section produisent, par ailleurs, plus de questions totales que celui de Petite Section. Cependant, des différences dans la répartition des questions posées apparaissent entre les deux types de support. Les enseignants de Petite Section et Grande Section produisent des questions partielles en plus grand nombre que pour la lecture sans texte et celui de Moyenne Section dans une moindre mesure. Les deux premiers enseignants émettent, en revanche, des questions totales et des questions ouvertes en moindre quantité alors que le dernier produit davantage de questions ouvertes. Parmi les questions ouvertes, les enseignants de Petite et Moyenne Section favorisent les questions en «pourquoi/comment» et les questions portant sur des événements et celui de Grande Section davantage les relances.

Les parents privilégient, quant à eux, les questions totales (ANA, JUL, LIL, JEA, LUD, ELI et OSC) et les questions partielles (JAM, ROM et SIM). D'un type de support à l'autre, les conduites des mères évoluent de par la proportion mais aussi la nature des questions posées. Les mères qui produisaient, pour la lecture sans texte, un grand nombre de questions partielles émettent désormais, mis à part la mère d'un enfant de 5 ans (SIM) qui ne modifie pas sa conduite, des questions totales dans une plus grande mesure. De plus, les mères qui produisaient des questions ouvertes ou totales utilisent désormais respectivement, mis à part la mère d'un enfant de 5 ans (OSC) qui ne change pas de conduite, des questions totales ou partielles. Par ailleurs, pour cette lecture avec texte, les mères des enfants de 3 ans émettent plus de questions en «pourquoi/comment» que pour la lecture sans texte. Ces mères mais aussi celles d'enfants âgés de 4 ans produisent plus de questions portant sur les événements que celles d'enfants âgés de 5 ans.

Ainsi, ces constats permettent de dégager différentes tendances.

Premièrement, il semblerait de nouveau que l'âge des enfants ait une influence sur les conduites des adultes. En effet, les enseignants et les parents ne privilégient pas le même type de questions (totales et ouvertes) parallèlement aux questions partielles selon l'âge des enfants.

Deuxièmement, il semblerait également que le type de support influe sur les conduites discursives des adultes. Même si les conduites des enseignants ne changent pas radicalement, puisqu'ils produisent en grande majorité des questions partielles, elles évoluent. La répartition des autres types de question change. Les mères paraissent, quant à elles, fortement influencées par le type de support. Il en ressort ainsi que la lecture sans texte permettrait d'avoir globalement moins de questions fermées dans les discours des adultes et favoriserait les questions partielles et/ou ouvertes. 
Les catégories de questions étant établies chez les différentes mères et enseignants, voyons quel rôle elles jouent dans l'étayage produit par les adultes et s'il existe, de la même façon que précédemment, une possible influence de l'âge des enfants et du type de support sur leurs conduites.

\subsection{Rôle des questions étayantes adressées aux enfants}

Le type d'étayage s'avère, comme nous l'avons déjà présenté, de deux types : linguistique ou impliqué dans la tâche.

\subsubsection{Types d'étayage dans la lecture sans texte et avec texte}

$\mathrm{Au}$ cours de chacune des lectures, quel que soit le support, les adultes effectuent le plus souvent un étayage de la tâche et dans une moindre mesure un étayage linguistique. Si nous observons de nouveau les exemples 17 et 18 présentés précédemment, nous constatons bien que l'enseignant de Petite Section et la mère de l'enfant de 4 ans délivrent essentiellement un étayage de la tâche à travers un fort guidage et peu d'étayage linguistique, une seule ébauche sur l'ensemble de l'étayage produit pour l'enseignant et une demande de dénomination pour la mère.

Des différences apparaissent cependant dans la répartition de ces différents types d'étayage des adultes selon l'âge des enfants ou le type de support. En effet, tant pour la lecture sans texte que celle avec texte, plus les enfants sont jeunes plus les enseignants adoptent un étayage de la tâche et plus les enfants sont âgés plus les enseignants produisent un étayage linguistique. Cela se traduit chez l'enseignant de Petite Section par un fort guidage de la narration et chez l'enseignant de Grande Section par la présence d'un étayage autre «autour» de la narration. Ainsi, l'enseignant de Grande Section réalise, en plus de la narration, d'autres types de discours.

Par ailleurs, les enseignants de Moyenne et Grande Section proposent aux enfants un étayage linguistique plus important dans la lecture avec texte et celui de Petite Section dans la lecture sans texte. Pour les mères, l'étayage linguistique semble davantage présent dans la lecture sans texte, avec les enfants les plus jeunes et les plus âgés, mais pas avec ceux de 4 ans, et inversement l'étayage de la tâche paraît davantage présent dans la lecture avec texte que dans celle sans texte. De plus, deux mères d'enfants âgés de 5 ans (ELI et OSC) voient leur répartition beaucoup évoluer d'un support à l'autre. Elles produisent nettement plus d'étayage de la tâche pour la lecture avec texte.

Voyons ce qu'il en est plus précisément pour chaque type d'étayage.

\subsubsection{L'étayage linguistique dans la lecture sans texte et avec texte}

Pour la lecture sans texte, l'enseignant de Petite Section produit un étayage linguistique essentiellement composé d'ébauches et de demandes de dénomination (comme nous avons pu le voir dans l'exemple 17) alors que les deux autres enseignants utilisent un étayage plus diversifié. Cet étayage de l'enseignant de Grande Section se traduit par une très forte présence d'ébauches mais aussi, dans une moindre mesure, de demandes de dénomination, de définition et de clarification alors que celui de l'enseignant de Moyenne Section se caractérise en très grande majorité par la présence de demandes de dénomination et dans une moindre mesure d'ébauches, de demandes de clarification et de dénomination.

Les conduites discursives des mères sont beaucoup plus hétérogènes. Même si une grande majorité des mères d'enfants âgés de 4 et 5 ans réalise des demandes de dénomination (JAM, JEA, LUD, ROM, ELI et $\mathrm{SIM}$ ), d'autres utilisent de manière quasi équivalente des demandes de dénomination mais aussi des demandes de définition (ANA) ou des ébauches (JUL et OSC). Une mère d'un enfant de 3 ans (LIL) ne produit aucun étayage linguistique. 
Pour la lecture avec texte, les enseignants de Petite Section et de Grande Section produisent essentiellement des ébauches mais aussi des demandes de dénomination; l'enseignant de Moyenne Section utilise en grande majorité des demandes de définition mais aussi des demandes de clarification et des ébauches. Le type de support engendre ainsi un effet sur les conduites des enseignants de Moyenne Section et Grande Section. L'étayage de l'enseignant de Grande Section se caractérise alors par une augmentation des ébauches et une diminution des demandes de dénomination et de définition; en revanche celui de Moyenne Section par une baisse des demandes de dénomination et une hausse des ébauches et des demandes de définition.

Les conduites discursives des mères pour ce support s'avèrent, comme pour la lecture sans texte, très hétérogènes. L'étayage linguistique de chacun d'entre elles est très peu diversifié. Une mère d'un enfant de 3 ans (LIL), une d'un enfant de 4 ans (JEA) et deux d'enfants de 5 ans (ELI et SIM) ne produisent qu'un type d'étayage qui correspond à des demandes de clarification, de définition ou de dénomination. Deux mères d'enfants âgés de 3 ans (JUL) et 4 ans (JAM) émettent de manière quasi équivalente des demandes de dénomination et respectivement des demandes de clarification et des ébauches. Seul le père d'un enfant de 4 ans (ROM) produit en grande majorité des ébauches. Le type de support semble donc également influer sur les conduites d'étayage linguistique des parents puisque leurs conduites s'en avèrent changées. Seule une mère (SIM) ne modifie pas son type d'étayage.

Ainsi, il en ressort également que non seulement le type de support peut avoir une incidence sur les conduites des adultes mais aussi l'âge des enfants, particulièrement pour la lecture sans texte.

Regardons ce qu'il en est de l'étayage spécifique de la tâche.

\subsubsection{L'étayage spécifique de la tâche dans la lecture sans texte et avec texte}

Pour la lecture sans texte, tant chez les enseignants que chez les mères, la conduite d'étayage de la tâche qui prédomine est la conduite de guidage. Cette conduite recoupe toutes les questions factuelles, les questions anticipatrices, les demandes d'explication et les relances. Ce type d'étayage s'avère plus présent chez l'enseignant de Petite Section que chez ceux de Moyenne et Grande Section; ils accordent cependant tous également une large part aux réactions aux productions des enfants ou aux conduites réflexives. Par ailleurs, les conduites réflexives semblent plus présentes chez une grande majorité des mères que chez les enseignants. L'implication dans la tâche parait, quant à elle, dépendre plus de diversités individuelles. Dans l'exemple 18, nous voyons que la mère produit, en plus du guidage, des demandes d'implication dans la tâche et à travers ses questions des reprises de propos de l'enfant.

Pour la lecture avec texte, le guidage occupe de la même façon une large part. L'enseignant de Petite Section accorde de nouveau une place plus importante à ce type d'étayage que les enseignants de Moyenne et Grande Section. Cependant, pour ce type de support, il semblerait qu'il y ait chez chacun d'entre eux une forte augmentation du guidage et une baisse des conduites réflexives et de l'implication dans la tâche. Les enseignants de Moyenne Section et Grande Section réagissent moins aux productions des enfants alors que celui de Petite Section y réagit davantage. Par ailleurs, chez quasiment toutes les mères (sauf chez celle d'un enfant de 4 ans JAM), à l'inverse des enseignants, il est possible de noter une décroissance du guidage et une augmentation des conduites réflexives et d'implication dans la tâche. Les conduites réflexives s'avèrent de nouveau plus présentes chez les parents que chez les enseignants. En revanche, les mères des enfants de 3 ans comme l'enseignant de Petite Section, réagissent davantage aux productions des enfants.

Ainsi, il semble qu'il y ait une incidence de l'âge sur ces conduites d'étayage de la tâche des enseignants alors qu'il ne parait pas y en avoir une sur celles des mères. De plus, il parait également y avoir une forte influence du type de support sur les conduites d'étayage des adultes. Les parents guident moins la lecture avec texte probablement du fait qui c'est un type de support qu'ils connaissent et maitrisent davantage que la lecture sans texte. 


\section{Conclusion}

Au vu de ces constats, il existe des similitudes mais aussi des différences dans les conduites des mères et des enseignants selon l'âge des enfants, le type de lecture, mais aussi selon les adultes entre eux.

Premièrement, de nombreuses similitudes apparaissent dans les conduites discursives de ces différents adultes. Les mères comme les enseignants produisent en grande majorité, quel que soit l'âge des enfants et le type de support, des assertions et des questions, un étayage de la tâche plus qu'un étayage linguistique et une conduite de guidage plus que les autres catégories d'étayage de la tâche.

Cependant, des différences notables émergent dans leurs conduites suivant leur statut. Quel que soit le type de support ou l'âge des enfants, les mères produisent plus d'assertions sollicitantes ou de conduites réflexives que les enseignants, qui réalisent, quant à eux, davantage d'ébauches. De plus, suivant l'âge des enfants, mères et enseignants peuvent adopter des conduites diamétralement opposées. L'enseignant de Petite Section comme les mères des enfants de 5 ans posent plus de questions (notamment ouvertes ou en "pourquoi/comment ») et utilisent moins d'assertions que l'enseignant de Grande Section et les mères des enfants de 3 ans qui émettent plus d'assertions et moins de questions. En plus de l'âge, la répartition du type d'étayage et des catégories d'étayage semblent varier chez les enseignants en fonction du type de support. Ces différences de conduites entre mères et enseignants peuvent être dues à leurs différences de visée de la tâche à accomplir. Comme le souligne Delamotte-Legrand (2012), la visée des parents est avant tout affective, ils seraient davantage à la recherche d'un plaisir partagé avec l'enfant. La visée des enseignants est avant tout éducative, liée à la question des apprentissages et des apports linguistiques. Cette visée des enseignants se retrouve particulièrement dans leurs différences de conduites discursives. Ceux-ci, de la Petite à la Grande Section, paraissent adapter leurs discours aux enfants qui se trouvent en face d'eux. Il pourrait donc exister une sorte de continuité entre les conduites des enseignants de la Petite Section à la grande Section, qui passent d'une conduite plus guidée de la narration à une conduite plus orientée vers la co-construction de la narration. Les mères se plaçant alors également sur cet axe dynamique plus en fonction de leur style particulier et moins de l'âge des enfants.

De plus, suivant le type de support, avec texte ou sans texte, les interventions verbales des enseignants mais aussi des mères peuvent changer. Trois types de conduites peuvent alors apparaitre: pas de modifications des interventions verbales, une modification de la répartition de ces interventions ou bien une inversion. Les enseignants se situent dans les deux premiers types de conduites alors que les mères peuvent adopter les trois.

Par ailleurs, les différentes conduites adoptées, par les enseignants en particulier mais aussi par certaines mères, montrent que la lecture de l'album sans texte demande un soutien, un guidage de la lecture plus marqué que pour la lecture avec texte.

Pour finir, bien que l'âge des enfants, le «statut » de l'adulte mais aussi, comme l'ont souligné Danis et al. (2000) et McGinty et al. (2012), les comportements et les conduites discursives des enfants, semblent influer sur les conduites discursives des mères et des enseignants, il semblerait également qu'il existe des variabilités individuelles qui puissent se regrouper en fonction de profils communs, comme l'ont remarqué Salazar Orvig et de Weck (à paraître), indépendants des premiers facteurs. Les enfants sont donc confrontés, tout au long de leur scolarité à l'école maternelle, à différents styles de conduites et d'expériences discursives qui leur permettront in fine de construire seuls des récits.

\section{Références bibliographiques}

Bignasca, T. \& Rezzonico, S. (2010). Étayage des mères et réactions des enfants dans une activité de narration, Langage \& pratiques, 46, 32-42.

Bruner, J.S. (1983). Le rôle de l'interaction de tutelle dans la résolution de problème. In J. Bruner (Ed.), Le développement de l'enfant: savoir faire, savoir dire. (pp. 261-279). Paris : Presses universitaires de France. 
Bruner, J. S. (1996). L'éducation, entrée dans la culture. Les problèmes de l'école à la lumière de la psychologie culturelle. Paris : Retz.

Bunting, E., \& Carpenter, N. (2005). Le bateau de Petit Ours. Paris : Lutin Poche de l'école des loisirs.

Bus, A. G., van Ijzendoorn, M. H., \& Pellegrini, A. D. (1995). Joint Book Reading Makes for Success in Learning to Read : A Meta-Analysison Intergenerational Transmission of Literacy, Review of Educational Research, 65(1), pp. 1-21.

Danis, A., Bernard, J.-M., \& Leproux, C. (2000). Shared picture-book reading : A sequential analysis of adult-child verbal interactions. British Journal of Developmental Psychology, 18(3), 369-388.

De Weck, G. (1998). Stratégies d'étayage avec des enfants dysphasiques : sont-elles spécifiques ?, TRANEL, 29, 1328.

Delamotte, R. \& Akinci, M.-A. (2012). Récits d'enfants. Développement, genre, contexte. Monts: Presse Universitaire de Rouen et du havre.

Florin, A. (1991). Pratiques du langage à l'école maternelle et prédiction de la réussite scolaire. Paris : PUF.

François, F., Hudelot, C., \& Sabeau-Jouannet, C. (1984). Conduites linguistiques chez le jeune enfant. Paris : PUF.

Goodall, J. S. (1980). Ah les belles vacances des petits cochons! Paris: Gallimard.

Grossmann, F. (1999). Littératie, compréhension et interprétation des textes, Repères, 19, 139-166.

Grossmann, F. (2001). Pour une approche ethnologique des activités de lecture : l'exemple des lecture partagées, Cahiers du français contemporains, 7, 135-160.

Hudelot, C. \& Vasseur, M. T. (1997). Peut-on se passer de la notion d'étayage pour rendre compte de l'élaboration langagière en L1 \& L2 ?, CAlap, 15, 109-135.

Ingold, J., Gendre, S., Rezzonico, S., Corlateanu, C., \& Da Silva, C. (2008). Diversité des étayages des mères d'enfants tout-venant et dysphasiques dans deux situations d'interaction. TRANEL (Travaux neuchâtelois de linguistique), 69-82.

Lafontaine, D. (1994). Quand les parents posent des questions à leurs jeunes enfants. Lafontaine, Enfance, 37 (1), 2140.

Low, J., \& Durkin, K. (2001). Individual differences and consistency in maternal talk style during joint story encoding and retrospection: Associations with children's long term recall. International Journal of Behavioral Development, 25, 27-36.

Mason, J. M., \& Allen, J. (1986). A review of emergent literacy with implications for research and practice in reading. Unpublished manuscript.

McGinty, A. S., Justice, L. M., Zucker, T. A., Gosse, C., \& Skibbe, L. E. (2012). Shared-Reading Dynamics : Mothers' Question Use and the Verbal Participation of Children With Specific Language Impairment. Journal of speech, language and hearing research, 55(4), 1039.

McNamee, G. D. (1987). The social origins of narrative skills. In M. Hickmann (Ed.), Social and functional approaches to language and thought (pp. 287-304). Orlando Fl.: Academic Press.

Nelson, K. (1991). Remembering and telling: A developmental story. Journal of Narrative and Life History, 1, 109127.

Ninio, A., \& Bruner, J. (1978). The achievement and antecedents of labelling. Journal of Child Language, 5, 1-15.

Pellegrini, A. D., Brody, G. H., \& Sigel, I. E. (1985a), Parents' book-reading habits with their children. Journal of Educational Psychology, 77, 332-340.

Pellegrini, A. D., Brody, G. H., \& Sigel, I. E. (1985b). Parents' teaching strategies with their children : The effects of parental and child status variables. Journal of Psycholinguistic Research, 14, 509-521.

Peterson, C., \& McCabe, A. (1994). A Social Interactionist Account of Developing Decontextualized Narrative Skill. Developmental Psychology, 30(6), 937-948.

Pratt, M.W., Kerig, P., Cowan, P.A., \& Cowan, C.P. (1988). Mothers and fathers teaching 3-year-olds: Authoritative 
parenting and adult scaffolding of young children's learning. Developmental Psychology, 24, 832-839.

Reese, E., \& Fivush, R. (1993). Parental styles of talking about the past. Developmental Psychology, 29, 596-606.

Rodriguez, B. (2008). Le voleur de poule. Paris : Editions Autrement.

Rondal J.A. (1978), Langage et éducation. Bruxelles : Mardaga.

Rondal J.A. (1983), L'interaction adulte-enfant et la construction du langage. Bruxelles : Mardaga.

Rosat, M.-C. (1998). Comparaison des stratégies discursives d'étayage dans un conte et un récit d'expériences oraux, TRANEL, 29, 29-47.

Salazar Orvig, A., \& de Weck, G. (à paraître). Profils de mères et implication des enfants dans la co-construction d'un récit, A.N.A.E., 124.

Sénéchal, M., Lefèvre, J. A., Thomas, E. M., \& Daley, K. E. (1998). Differential Effects of Home Literacy Experiences on the Development of Oral and Written, Reading Research Quarterly, 33 (1), 96-116.

Snow, C.E. (1983). Literacy and language: Relationships during the preschool years. Harvard Educational Review, $53,165-189$.

Snow, C.E., \& Ninio, A. (1986). The contracts of Literacy : What Children Learn from Learnong to Read Books. In W.H. Teale \& E. Sulzby (Eds.), Emergent literacy: Writing and reading (pp.116-138). Norwood, NJ : Ablex.

Sulzby, E. (1985). Children's emergent reading of favorite storybooks: A developmental study. Reading Research Quarterly, 20, 458-481.

Sulzby, E., \& Teale, W. (1991). Emergent literacy. In R. Barr et al. (Eds.), Handbook of reading research (Vol. 2, pp. 727-758). New York : Longman.

Teale, W. H., \& Sulzby, E. (1986). Emergent literacy as a perspective for examining how young children become writers and read-ers. In W.H. Teale \& E. Sulzby (Eds.), Emergent literacy: Writing and reading (pp. vii-xxv). Norwood, NJ : Ablex.

Thomas, B. (1984). Early toy preferences of four-year-old readers and nonreaders. Child Development, 55, 424-430.

Vygotski, L. S. (1997). Pensée et langage. Paris: La Dispute.

Wadell, M, \& Firth, B. (1996). Toi et moi, Petit Ours. Paris : Lutin Poche de l'école des loisirs.

Wadell, M, \& Firth, B. (2005). La grotte de Petit Ours. Paris : Pastel de l'école des loisirs.

\footnotetext{
${ }^{1}$ ce corpus s'inscrit dans un travail de thèse et dans le cadre de la recherche «Acquisition des expressions référentielles en dialogue : approches multidimensionnelles (DIAREF) » financée par l'ANR dans le programme «Enfants-Enfance » (ANR 09- ENFT - 055) dirigé par Anne Salazar Orvig

2 childes.psy.cmu.edu/clan/

3 projet FNRS suisse «Interactions mère-enfant en situation logopédique » (subside no 100012-111938) dirigé par Geneviève de Weck en collaboration avec Anne Salazar Orvig
} 